William D. L. Appling, Mathematics Department, University of North

Texas, Denton, TX 76203

\title{
A CHARACTERIZATION THEOREM FOR THE EXISTENCE OF A HELLINGER-TYPE INTEGRAL
}

\begin{abstract}
Suppose that $a<b$ and each of $h$ and $m$ is a real-valued function defined on $[a ; b]$ with $m$ nondecreasing such that if $[p ; q] \subseteq[a ; b]$ and $\left.m\right|_{p} ^{q}=0$, then $\left.h\right|_{p} ^{q}=0$. There are developed, among other things, necessary and sufficient conditions in order that for each real-valued function $f$ defined and quasi-continuous on $[a ; b]$, the Hellinger - type integral

$$
\int_{[a ; b]} \frac{d f d h}{d m}
$$

exists. As is well known, this integral has arisen in connection with, among other things, representation theorems for certain classes of continuous linear functionals.
\end{abstract}

\section{Introduction}

Suppose that $a<b$. Previous authors [3], [5], [6], [7] have treated the representation of the elements of particular classes of linear functionals, complexvalued, defined on the space of complex-valued functions quasi-continuous on $[a ; b]$, and satisfying certain continuity conditions. In certain of these investigations, a Hellinger [2]-type integral, a limit of sums for subdivison refinement (see below and Section 2),

$$
\int_{[a ; b]} \frac{d f d h}{d m}
$$

has arisen, where $f$ belongs to the aforementioned space, and $h$ and $m$ are special real-valued functions defined on $[a ; b]$ with $m$ nondecreasing.

Key Words: integral existence characterization, bounded difference quotient variation, convex decomposition

Mathematical Reviews subject classification: Primary: 28A25

Received by the editors February 1, 1996 
We shall confine our attention in this paper to real-valued functions and pursue an existence characterization question that arises naturally in connection with the above integral. We develop results that, among other things, imply Theorem 1.1, which we state below following some preliminary remarks.

In order to make matters in this section more accessible and explicit, we digress and give some preliminary material, mainly definitions, that ordinarily would be in the initial part of Section 2. We label such things accordingly.

We adopt the convention that if each of $x$ and $y$ is in $\mathbb{R}$, then $\frac{x}{y}=0$ if $y=0$, and has the usual meaning otherwise.

Definition 2.1 Suppose that $u<v$. Then:

1) $\mathbb{R}[u ; v]$ is the set of all functions $f$ such that $[u ; v] \subseteq \operatorname{Dom}(f)$ and $\operatorname{Rng}(f) \subseteq \mathbb{R}$.

2) $\mathbb{R S I}[u ; v]$ is the set of all functions $A$ such that $\{[p ; q]: u \leq p<q \leq v\}$ $\subseteq \operatorname{Dom}(A)$ and $\operatorname{Rng}(A) \subseteq \mathbb{R}$.

3) $\exp (\mathbb{R}) \operatorname{SI}[u ; v]$ is the set of all functions $A$ such that $\{[p ; q]: u \leq p<$ $q \leq v\} \subseteq \operatorname{Dom}(A)$ and $\operatorname{Rng}(A) \subseteq \exp (\mathbb{R})$.

4) $\mathrm{C}[u ; v]$ is the set of all elements of $\mathbb{R}[u ; v]$ continuous on $[u ; v]$.

5) $\mathrm{C}_{0}[u ; v]$ is the set of all elements $f$ of $\mathrm{C}[u ; v]$ for which $f(u)=0$.

6) $\mathrm{BV}[u ; v]$ is the set of all elements of $\mathbb{R}[u ; v]$ having bounded variation on $[u ; v]$.

7) if $m$ is in $\mathbb{R}[u ; v]$ and is nondecreasing on $[u ; v]$, then $\operatorname{Lip}(m)[u ; v]$ is the set of all elements $h$ of $\mathbb{R}[u ; v]$ such that for some $K \geq 0$, if $u \leq p<q \leq v$, then $|h(q)-h(p)| \leq K(m(q)-m(p))$.

Definition 2.2 Suppose that $u<v$. Then:

1) The statement that $D$ is a subdivision of $[u ; v]$ means that $D$ is a finite collection of nonoverlapping intervals whose union is $[u ; v]$.

2) If $E$ is a subdivision of $[u ; v]$, then the statement that $H$ is a refinement of $E$, written, when appropriate as " $H \ll E$ " means that $H$ is a subdivision of $[u ; v]$ such that each element of $H$ is a subset of some element of $E$.

Definition 2.3 Suppose that $\alpha$ is a function whose range is a collection of sets. The statement that $b$ is an $\alpha$-function on $S$ means that $b$ is a function, $\operatorname{Dom}(b)=S \subseteq \operatorname{Dom}(\alpha)$, and for each $x$ in $S, b(x)$ is in $\alpha(x)$.

Definition 2.4 Suppose that $u<v$ and $\alpha$ is in $\exp (\mathbb{R}) \operatorname{SI}[u ; v]$. The statement that $K$ is an integral of $\alpha$ on $[u ; v]$ means that $K$ is in $\mathbb{R}$, and if $0<c$, then there is $D \ll\{[a ; b]\}$ such that if $E \ll D$ and $b$ is an $\alpha$-function on $E$, then

$$
\left|K-\sum_{E} b(I)\right|<c .
$$

Basic Uniqueness Assertion and Notation. Given the setting of Definition 2.4, if each of $K_{1}$ and $K_{2}$ is an integral of $\alpha$ on $[u ; v]$, then $K_{1}=K_{2}$; 
this element of $\mathbb{R}$ shall be called the integral of $\alpha$ on $[u ; v]$ and shall be denoted by $\int_{[u ; v]} \alpha(I)$ or $\int_{[u ; v]} \alpha$, depending on circumstances. We also adopt the convention that the statement " $\int_{[u ; v]} \alpha(I)$ exists" or " $\int_{[u ; v]} \alpha$ exists" shall mean that some $K$ is the integral of $\alpha$ on $[u ; v]$.

Finite Additivity Fact. Suppose that $s \leq p<q<r \leq t$ and $\alpha$ is in $\exp (\mathbb{R}) \mathrm{SI}[s ; t]$. If each of $\int_{[p ; q]} \alpha$ and $\int_{[q ; r]} \alpha$ exists, then $\int_{[p ; r]} \alpha$ exists and is $\int_{[p ; q]} \alpha+\int_{[q ; r]} \alpha$.

Our discussion of further basic properties of subdivisions, sums and integrals will be continued in Section 2 .

We resume our introduction with the theorem, mentioned above, that summarizes and follows from the main separate results of this paper. Note, with regard to statement 5), the roll of convexity.

Theorem 1.1 Suppose that each of $h$ and $m$ is a real-valued function defined on $[a ; b]$ with $m$ nondecreasing and that if $a \leq p<q \leq b$ and $m(q)-m(p)=0$, then $h(q)-h(p)=0$. The following four statements are equivalent:

1) If $f$ is a real-valued function defined and quasi-continuous on $[a ; b]$, then

$$
\int_{[a ; b]} \frac{d f d h}{d m}
$$

exists (see Section 2 for our division convention as well as the notions and notations below),

2) If $f$ is a real-valued function defined and quasi-continuous on $[a ; b]$, then for some subdivision $D$ of $[a ; b], K \geq 0$, and every refinement $E$ of $D$,

$$
\left|\sum_{E} \frac{(f(q)-f(p))(h(q)-h(p))}{m(q)-m(p)}\right| \leq K,
$$

3) If $f$ is a real-valued function defined and continuous on $[a ; b]$, then for some subdivision $D$ of $[a ; b], K \geq 0$, and every refinement $E$ of $D$,

$$
\left|\sum_{E} \frac{(f(q)-f(p))(h(q)-h(p))}{m(q)-m(p)}\right| \leq K,
$$

4) $h$ is in $\operatorname{Lip}(m)[a ; b]$ and "has bounded difference quotient variation with respect to $m$ on $[a ; b]$ ", i.e., for some $M \geq 0$, nondegenerate subdivision $D$ of $[a ; b]$ and each refinement $E$ of $D$,

$$
\sum_{E^{o}}\left|\frac{h(q)-h(p)}{m(q)-m(p)}-\frac{h\left(q^{\prime}\right)-h\left(p^{\prime}\right)}{m\left(q^{\prime}\right)-m\left(p^{\prime}\right)}\right| \leq M
$$


where $[a ; y]$ is in $E, E^{o}=E-\{[a ; y]\}$ and for each $[p ; q]$ in $E^{o},\left[p^{\prime} ; q^{\prime}\right]$ is in $E$ and $q^{\prime}=p$, and

5) there are $f_{1}$ and $f_{2}$, each in $\operatorname{Lip}(m)[a ; b]$, such that:

a) if $a \leq x \leq b$, then $f_{1}(x)-f_{2}(x)=h(x)-h(a)$,

b) if $i=1,2$, then

i) for some $M_{i} \geq 0$, nondegenerate subdivision $D_{i}$ of $[a ; b]$ and each refinement $E$ of $D_{i}$,

$$
\sum_{E^{o}}\left|\frac{f_{i}(q)-f_{i}(p)}{m(q)-m(p)}-\frac{f_{i}\left(q^{\prime}\right)-f_{i}\left(p^{\prime}\right)}{m\left(q^{\prime}\right)-m\left(p^{\prime}\right)}\right| \leq M_{i},
$$

notation as in 4) above, and

ii) if $i=1,2$, then $f_{i}$ is " $m$ convex upward on $[a ; b]$ ", i.e., if $a \leq t<$ $u \leq w<v \leq b$ and $0<\min \left\{\left.m\right|_{t} ^{u},\left.m\right|_{w} ^{v}\right\}$, then

$$
\frac{f_{i}(u)-f_{i}(t)}{m(u)-m(t)} \leq \frac{f_{i}(v)-f_{i}(w)}{m(v)-m(w)} .
$$

Now, it is obvious that in Theorem 1.1, 1) immediately implies 2) and 2) immediately implies 3 ). So the pattern that we will follow will be to show that 3) implies 4), 4) implies 1), 4) implies 5) and 5) implies 4). Accordingly, in Sections 3, 4 and 5, respectively, we prove Theorems 3.3, 4.8, and 5.4, stated below; clearly, the first of these is a generalization of the assertion that 3) implies 4), the second states that 4) implies 1), and the third states the equivalence of 4) and 5).

Theorem 3.3 Suppose that $a<b$ and $A$ is a real-valued function defined on the set of subintervals of $[a ; b]$ such that if $f$ is a real-valued function defined and continuous on $[a ; b]$, then there is a subdivision $D$ of $[a ; b]$ such that

$$
\left\{\sum_{E}(f(q)-f(p)) A[p ; q]: \text { E a refinement of } D\right\}
$$

is bounded. Then there is a nondegenerate subdivision $H$ of $[a ; b]$ and $M \geq 0$ such that if $E$ is a refinement of $H,[a ; r]$ is in $E, E^{o}=E-\{[a ; r]\}$, and for each $[p ; q]$ in $E^{o},\left[p^{\prime} ; q^{\prime}\right]$ is in $E$ and $q^{\prime}=p$, then

$$
\sum_{E^{o}}\left|A[p ; q]-A\left[p^{\prime} ; q^{\prime}\right]\right| \leq M
$$

and if $[p ; q]$ is in $E$, then

$$
|A[p ; q]| \leq M
$$


Theorem 4.8 Suppose that $a<b$, each of $h, m$, and $w$ is a real-valued function defined on $[a ; b], M \geq 0, w$ is quasi-continuous on $[a ; b], m$ is nondecreasing on $[a ; b], h$ is in $\operatorname{Lip}(m)[a ; b]$ and for some nondegenerate subdivision $D$ of $[a ; b]$, for each refinement $E$ of $D$ (see Theorem 3.3 above for notation)

$$
\sum_{E^{o}}\left|\frac{h(q)-h(p)}{m(q)-m(p)}-\frac{h\left(q^{\prime}\right)-h\left(p^{\prime}\right)}{m\left(q^{\prime}\right)-m\left(p^{\prime}\right)}\right| \leq M
$$

Then

$$
\int_{[a ; b]} \frac{d w d h}{d m}
$$

exists.

Theorem 5.4 Suppose that $a<b$, each of $h$ and $m$ is a real-valued function defined on $[a ; b]$ with $m$ nondecreasing on $[a ; b]$. Then the following two statements are equivalent:

1) $h$ is in $\operatorname{Lip}(m)[a ; b]$ and, for some $M \geq 0$, nondegenerate subdivision $D$ of $[a ; b]$ and each refinement $E$ of $D$,

$$
\sum_{E^{o}}\left|\frac{h(q)-h(p)}{m(q)-m(p)}-\frac{h\left(q^{\prime}\right)-h\left(p^{\prime}\right)}{m\left(q^{\prime}\right)-m\left(p^{\prime}\right)}\right| \leq M,
$$

where $[a ; y]$ is in $E, E^{o}=E-\{[a ; y]\}$ and for each $[p ; q]$ in $E^{o},\left[p^{\prime} ; q^{\prime}\right]$ is in $E$ and $q^{\prime}=p$.

2) There are $f_{1}$ and $f_{2}$, each in $\operatorname{Lip}(m)[a ; b]$, such that

a) if $a \leq x \leq b$, then $f_{1}(x)-f_{2}(x)=h(x)-h(a)$,

b) if $i=1,2$, then

i) for some $M_{i} \geq 0$, nondegenerate subdivision $D_{i}$ of $[a ; b]$ and each refinement $E$ of $D_{i}$,

$$
\sum_{E^{o}}\left|\frac{f_{i}(q)-f_{i}(p)}{m(q)-m(p)}-\frac{f_{i}\left(q^{\prime}\right)-f_{i}\left(p^{\prime}\right)}{m\left(q^{\prime}\right)-m\left(p^{\prime}\right)}\right| \leq M_{i},
$$

notation as in 1) above, and

ii) if $i=1,2$, then $f_{i}$ is " $m$ convex upward on $[a ; b]$ ", i.e., if $a \leq t<$ $u \leq w<v \leq b$ and $0<\min \left\{\left.m\right|_{t} ^{u},\left.m\right|_{w} ^{v}\right\}$, then

$$
\frac{f_{i}(u)-f_{i}(t)}{m(u)-m(t)} \leq \frac{f_{i}(v)-f_{i}(w)}{m(v)-m(w)} .
$$

We end this section with the remark that Theorem 1.1, some fairly simple integral equivalence results, a continuous function approximation theorem and integration by parts lead to a proof of the "classic" form of the Riesz Representation Theorem that does not require the use of certain well-known theorems of Helly. Space limitations preclude our inclusion of this development. 


\section{Preliminary Theorems and Definitions}

We now resume our discussion of preliminary matters begun in the introduction.

Definition 2.5 Suppose that $u<v$ and $\alpha$ is in $\exp (\mathbb{R}) \operatorname{SI}[u ; v]$. If $u \leq p<$ $q \leq v$, then the statement that $\alpha$ is $\sum$-bounded on $[p ; q]$ with respect to $D$ means that $D \ll\{[u ; v]\}$ and

$$
\left\{\sum_{E} b(I): E \ll\{[p ; q]\}, E \subseteq H \ll D\right\}
$$

is bounded; furthermore,

$$
L_{D}(\alpha)[p ; q] \text { and } G_{D}(\alpha)[p ; q]
$$

denote, respectively, the sup and inf of the above mentioned set.

Theorem 2.A.1 Suppose that $u<v, \alpha$ is in $\exp (\mathbb{R}) \operatorname{SI}[u ; v], u \leq p<q \leq v$ and $\alpha$ is $\sum$-bounded on $[p ; q]$ with respect to $D$ (we omit the subscript for $L$ and $G$ in the assertions that follow). Then:

1) If $p \leq r<s \leq q$, then $\alpha$ is $\sum$-bounded on $[r ; s]$ with respect to $D$.

2) If $D_{3} \ll D_{1} \ll\{[p ; q]\}$ and $D_{3} \ll D_{2} \ll\{[p ; q]\}$, then

$$
\sum_{D_{1}} G(\alpha)\left(I_{1}\right) \leq \sum_{D_{3}} G(\alpha)\left(I_{3}\right) \leq \sum_{D_{3}} L(\alpha)\left(I_{3}\right) \leq \sum_{D_{2}} L(\alpha)\left(I_{2}\right),
$$

so that the following integrals exist and the following inequality holds:

$$
\int_{[p ; q]} G(\alpha) \leq \int_{[p ; q]} L(\alpha)
$$

3) $\int_{[p ; q]} \alpha$ exists iff $\int_{[p ; q]} G(\alpha)=\int_{[p ; q]} L(\alpha)$,, in which case

$$
\int_{[p ; q]} G(\alpha)=\int_{[p ; q]} \alpha=\int_{[p ; q]} L(\alpha) .
$$

We now state a theorem which is essentially Kolmogoroff's [4] Differential Equivalence Theorem for the setting of this paper.

Theorem 2.K.1 Suppose that $u<v, \alpha$ is in $\exp (\mathbb{R}) \operatorname{SI}[u ; v], u \leq p<q \leq v$ and $\int_{[p ; q]} \alpha$ exists. Then:

1) if $p \leq r<s \leq q$, then $\int_{[r ; s]} \alpha$ exists, and 
2) the following existence and equality assertion holds:

$$
\int_{[p ; q]}\left|\alpha(I)-\int_{I} \alpha\right|=0
$$

i.e., if $0<c$, then there is $D \ll\{[p ; q]\}$ such that if $E \ll D$ and $b$ is an $\alpha$-function on $E$, then

$$
\sum_{E}\left|b(I)-\int_{I} \alpha\right|<c
$$

We now discuss some integral existence and equivalence properties that shall use in Section 4 (also see [1]).

Theorem 2.A.2 The following statements are true:

1) Suppose that each of $\beta$ and $\gamma$ is in $\mathbb{R S I}[a ; b]$ and is interval-wise additive on $[a ; b]$, i.e., if $\delta$ is $\beta$ or $\gamma$ and $a \leq p<q<r \leq b$, then $\delta[p ; q]+\delta[q ; r]=\delta[p ; r]$. The following statements hold:

a) If $a \leq u<v \leq b$ and for $i=1,2, E \ll D \ll\{[u ; v]\}$, then

$$
\begin{aligned}
\sum_{E_{1}} \min \{\beta(J), \gamma(J)\} & \leq \sum_{D} \min \{\beta(I), \gamma(I)\} \\
& \leq \sum_{D} \max \{\beta(I), \gamma(I)\} \leq \sum_{E_{2}} \max \{\beta(J), \gamma(J)\}
\end{aligned}
$$

b) From a) it follows that if $a \leq u<v \leq b$, then $\int_{[u ; v]} \min \{\beta, \gamma\}$ exists iff $\left\{\sum_{H} \min \{\beta(J), \gamma(J)\}: H \ll\{[u ; v]\}\right\}$ is bounded below, and $\int_{[u ; v]} \max \{\beta, \gamma\}$ exists iff $\left\{\sum_{H} \max \{\beta(J), \gamma(J)\}: H \ll\{[u ; v]\}\right\}$ is bounded above.

2) If $a \leq u<v \leq b$, each of $A$ and $B$ is in $\exp (\mathbb{R}) \operatorname{SI}[u ; v]$, each of $\int_{[u ; v]} A$ and $\int_{[u ; v]} B$ exists and $Q$ is $\max$ or min, then, by Theorem 2.K.1, (see [1] again)

$$
\int_{[u ; v]}\left|Q\{A(I), B(I)\}-Q\left\{\int_{I} A, \int_{I} B\right\}\right|=0,
$$

so that if $u \leq p<q \leq v$, then

$$
\int_{[p ; q]} Q\{A(I), B(I)\} \text { exists iff } \int_{[p ; q]} Q\left\{\int_{I} A, \int_{I} B\right\} \text { exists, }
$$

in which case equality holds.

3) From 2) it follows that if $a \leq u<v \leq b$ and $C$ is in $\exp (\mathbb{R}) \operatorname{SI}[u ; v]$ and $\int_{[u ; v]} C$ exists, then

$$
\int_{[u ; v]}|C(I)|-\left|\int_{I} C\right| \mid=0
$$


so that if $u \leq p<q \leq v$, then

$$
\int_{[p ; q]}|C(I)| \text { exists iff } \int_{[p ; q]}\left|\int_{I} C\right| \text { exists },
$$

in which case equality holds.

4) From 1b) it follows that if $\beta$ and $\gamma$ are further such that if $\delta$ is $\beta$ or $\gamma$, then $\left\{\sum_{H}|\delta(J)|: H \ll\{[u ; v]\}\right\}$ is bounded and $Q$ is $\max$ or min, then $\int_{[u ; v]} Q\{\beta, \gamma\}$ exists.

\section{Consequences of Certain Kinds of $\Sigma$-boundedness}

In this section we prove Theorem 3.5, as stated in the introduction

Notation. (the usual $\Delta$-notation) If $r<s$ and $z$ is a real-valued function defined on $[r ; s]=I$, then $\Delta_{I} z=z(s)-z(r)$.

Theorem 3.1 Suppose that $a<b, A$ is in $\mathbb{R S I}[a ; b]$ and for each $f$ in $C[a ; b]$ there is $D \ll[a ; b]$ such that

$$
\left\{\sum_{E} \Delta_{I} f A(I): E \ll D\right\}
$$

is bounded. It follows that if $a<w \leq b$, then for some $u$ such that $a \leq u<w$, $\{A[v ; w]: u \leq v<w\}$ is bounded, and if $a \leq w<b$, then for some $v$ such that $w<v \leq b,\{A[w ; y]: w<y \leq v\}$ is bounded.

Proof. We show the first part of the conclusion; the second part follows similarly.

Suppose the contrary. Then, for some increasing sequence $\left\{u_{k}\right\}_{k=1}^{\infty}$ of numbers of $[a ; w], u_{n} \rightarrow w$ and $\left|A\left[u_{n} ; w\right]\right| \rightarrow \infty, n \rightarrow \infty$. There are subsequences, $\left\{p_{i}\right\}_{i=1}^{\infty}$ and $\left\{q_{i}\right\}_{i=1}^{\infty}$ of $\left\{u_{k}\right\}_{k=1}^{\infty}$ such that for all $i, p_{i}<q_{i}<p_{i+1}<q_{i+1}$ and for all $\mathrm{n}$ in $\mathbb{P}$,

$$
\left|A\left[p_{n+1} ; w\right] \sum_{j=n+1}^{\infty} 2^{-j}\right|>n+1+\sum_{i=1}^{n}\left|A\left[p_{i} ; q_{i}\right] 2^{-i}\right| .
$$

There is an element $g$ of $\mathrm{C}[a ; b]$ such that for all $m$ in $\mathbb{P}$,

$$
g(x)= \begin{cases}\sum_{j=1}^{\infty} 2^{-j} & \text { if } a \leq x \leq p_{1} \\ \sum_{j=m}^{\infty} 2^{-j} & \text { if } x=p_{m} \\ \sum_{j=m+1}^{\infty} 2^{-j} & \text { if } q_{m} \leq x \leq p_{m+1} \\ 0 & \text { if } w \leq x \leq b\end{cases}
$$


Now, suppose that $D \ll\{[a ; b]\}$ and $0<K$. There is $E \ll D$ such that for some $n^{*}$ in $\mathbb{P},\left[p_{n^{*}} ; w\right]$ is in $E$. There is $W$ in $\mathbb{P}$ such that

$$
W>\max \left\{n^{*}+1, K+\left|\sum_{E-\left\{\left[p_{\left.\left.n^{*} ; w\right]\right\}}\right.\right.} A(I) \Delta_{I} g\right|\right\}
$$

Let

$$
H=\left(E-\left\{\left[p_{n^{*}} ; w\right]\right\}\right) \cup\left\{\left[p_{n^{*}} ; q_{n^{*}}\right],\left[q_{n^{*}} ; p_{n^{*}+1}\right],\left[p_{n^{*}+1} ; q_{n^{*}+1}\right], \ldots,\left[p_{W} ; w\right]\right\}
$$

Then $H \ll E$ and

$$
\begin{aligned}
& \left|\sum_{H} A(I) \Delta_{I} g\right| \\
& =\left|A\left[p_{W} ; w\right] \sum_{j=W}^{\infty}\left(-2^{-j}\right)+\sum_{i=n^{*}}^{W-1} A\left[p_{i} ; q_{i}\right]\left(-2^{-i}\right)+\sum_{E-\left\{\left[p_{n^{*}} ; w\right]\right\}} A(I) \Delta_{I} g\right| \\
& \geq\left|A\left[p_{W} ; w\right] \sum_{j=W}^{\infty} 2^{-j}\right|-\sum_{i=n^{*}}^{W-1}\left|A\left[p_{i} ; q_{i}\right] 2^{-i}\right|-\left|\sum_{E-\left\{\left[p_{n^{*}} ; w\right]\right\}} A(I) \Delta_{I} g\right| \\
& >W+\sum_{i=1}^{W-1}\left|A\left[p_{i} ; q_{i}\right] 2^{-i}\right|-\sum_{i=n^{*}}^{W-1}\left|A\left[p_{i} ; q_{i}\right] 2^{-i}\right|-\left|\sum_{E-\left\{\left[p_{n^{*}} ; w\right]\right\}} A(I) \Delta_{I} g\right| \\
& \geq W-\left|\sum_{E-\left\{\left[p_{\left.n^{*} ; w\right]}\right.\right.} A(I) \Delta_{I} g\right|>K,
\end{aligned}
$$

a contradiction.

Therefore the conclusion of the theorem is true.

Notational device. If, in a given discussion, an expression of substantial complexity is to be written more than once, it will be enclosed in brackets and a subscript affixed; thereafter only the brackets with subscript need be written.

Theorem 3.2 Suppose the hypothesis of Theorem 3.1. Then there is $D \ll$ $\{[a ; b]\}$ such that $\{A(I): I$ in $H \ll D\}$ is bounded.

Proof. Suppose the contrary. Then there is a set, $M$, such that $x$ is in $M$ iff $a<x \leq b$ and if $D \ll\{[a ; x]\}$, then $\{A(I): I$ in $H \ll D\}$ is unbounded. Let $z$ denote $\inf M . a \leq z$ and $z$ is in $M$ or not.

Suppose that $z$ is in $M$. Then $a<z$. Suppose that $a<y<z$. Then there is $E \ll\{[a ; y]\}$ such that $\{A(I): I$ in $H \ll E\}$ is bounded. Suppose that 
$P \ll\{[y ; z]\}$. Since $\{A(I): I$ in $H \ll E \cup P\}$ is unbounded, it follows that $\{A(I): I$ in $H \ll P\}$ is unbounded.

Suppose that $z$ is not in $M$. Then $z<b$. Suppose that $z<y<b$. There is $t$ in $M$ such that $t<y . z<t<y$. If $D \ll\{[a ; t]\}$, then $\{A(I): I$ in $H \ll D\}$ is unbounded. If $z=a$, then, if $D \ll\{[z ; t]\}$, then $\{A(I): I$ in $H \ll D\}$ is unbounded. So suppose that $a<z$. Then there is $E \ll\{[a ; z]\}$ such that $\{A(I): I$ in $H \ll E\}$ is bounded. Suppose that $Q \ll\{[z ; t]\}$. Then $\{A(I): I$ in $H \ll E \cup Q\}$ is unbounded, so that $\{A(I): I$ in $H \ll Q\}$ is unbounded, so that if $P \ll\{[z ; y]\}$, then $\{A(I): I$ in $H \ll P\}$ is unbounded.

Therefore there is $z$ in $[a ; b]$ such that either:

i) $z<b$ and if $z<y<b$ and $D \ll\{[z ; y]\}$, then $\{A(I): I$ in $H \ll D\}$ is unbounded, or

ii) $a<z$ and if $a<y<z$ and $D \ll\{[y ; z]\}$, then $\{A(I): I$ in $H \ll D\}$ is unbounded.

We shall assume i); the conclusion of the theorem follows from ii) similarly. Now, it follows from Theorem 3.1 that there is $K^{*} \geq 0$ and $s$ such that $z<s \leq$ $b$ such that if $z<r \leq s$, then $|A[z ; r]| \leq K^{*}$. Now, suppose that $K^{*}<K^{\prime}$, $z<t \leq s$ and $D \ll\{[z ; t]\}$. Since $\{A(I): I$ in $H \ll D\}$ is unbounded, it follows that for some $[p ; q] \subseteq$ some element $W$ of $D, K^{\prime}<|A[p ; q]|$. Clearly $p \neq z$, so $z<p<q \leq t$.

It follows that there is a sequence $\left\{\left[p_{i} ; q_{i}\right]\right\}_{i=1}^{\infty}$ of intervals such that for all $i$ in $\mathbb{P}$,

$$
z<p_{i+1}<q_{i+1} \leq p_{i}<q_{i} \leq s, q_{n} \rightarrow z, n \rightarrow \infty
$$

and for all $n$,

$$
\left|2^{-(n+1)} A\left[p_{n+1} ; q_{n+1}\right]\right|>n+\sum_{i=1}^{n}\left|2^{-i} A\left[p_{i} ; q_{i}\right]\right| .
$$

Routine considerations tell us that there is an element $g$ of $\mathrm{C}[a ; b]$ such that if $i$ is in $\mathbb{P}$, then

$$
g\left(q_{i}\right)-g\left(p_{i}\right)=2^{-i} \text { and } g\left(p_{i}\right)-g\left(q_{i+1}\right)=0 .
$$

Now, suppose that $D \ll\{[a ; b]\}$ and $0<K$. There is $E \ll D$ such that for some $n^{*}$ in $\mathbb{P},\left[z ; q_{n^{*}}\right]$ is in $E$. There is $W$ in $\mathbb{P}$ such that

$$
\begin{aligned}
& W>n^{*}+1 \text { and } W>K \\
& \quad+K^{*} \sup \left\{\left|\Delta_{I} g\right|: I \text { subinterval of }[a ; b]\right\}+\left|\sum_{E-\left\{\left[z ; q_{n^{*}}\right]\right\}} A(I) \Delta_{I} g\right| .
\end{aligned}
$$

Let

$$
H=\left(E-\left\{\left[z ; q_{n^{*}}\right]\right\}\right) \cup\left\{\left[z ; p_{W}\right],\left[p_{W} ; q_{W}\right], \ldots,\left[p_{n^{*}} ; q_{n^{*}}\right]\right\}
$$


Clearly $H \ll E$ and

$$
\begin{aligned}
& \left|\sum_{H} A(I) \Delta_{I} g\right| \\
& =\left|A\left[z ; p_{W}\right] \Delta_{\left[z ; p_{W}\right]} g+\sum_{i=n^{*}}^{W} A\left[p_{i} ; q_{i}\right] 2^{-i}+\sum_{E-\left\{\left[z ; q_{n^{*}}\right]\right\}} A(I) \Delta_{I} g\right| \\
& =\mid A\left[p_{W} ; q_{W}\right] 2^{-W}+\sum_{i=n^{*}}^{W-1} A\left[p_{i} ; q_{i}\right] 2^{-i}+A\left[z ; p_{W}\right] \Delta_{\left[z ; p_{W}\right]} g \\
& +\sum_{E-\left\{\left[z ; q_{n^{*}}\right]\right\}} A(I) \Delta_{I} g \\
& \geq\left|A\left[p_{W} ; q_{W}\right] 2^{-W}\right|-\sum_{i=n^{*}}^{W-1}\left|A\left[p_{i} ; q_{i}\right] 2^{-i}\right|-\left|A\left[z ; p_{W}\right]\right|\left|\Delta_{\left[z ; p_{W}\right]} g\right| \\
& -\left|\sum_{E-\left\{\left[z ; q_{n^{*}}\right]\right\}} A(I) \Delta_{I} g\right| \\
& >W+\sum_{i=1}^{W-1}\left|A\left[p_{i} ; q_{i}\right] 2^{-i}\right|-\sum_{i=n^{*}}^{W-1}\left|A\left[p_{i} ; q_{i}\right] 2^{-i}\right| \\
& -K^{*} \sup \left\{\left|\Delta_{I} g\right|: I \text { subinterval of }[a ; b]\right\}-\left|\sum_{E-\left\{\left[z ; q_{n^{*}}\right]\right\}} A(I) \Delta_{I} g\right| \\
& >K+\left[K^{*} \sup \left\{\left|\Delta_{I} g\right|: I \text { subinterval of }[a ; b]\right\}+\left|\sum_{E-\left\{\left[z ; q_{n^{*}}\right]\right\}} A(I) \Delta_{I} g\right|\right]_{1} \\
& -[]_{1}=K,
\end{aligned}
$$

a contradiction of the hypothesis.

Therefore the conclusion of the theorem is true.

Before continuing, we introduce some notation and make some elementary observations.

Definition 3.1 Suppose that $u<v, D \ll\{[u ; v]\}$ and $D$ contains two elements. Then $L_{D}$ denotes $[u ; t]$ in $D, R_{D}$ denotes $[w ; v]$ in $D$, for each $[p ; q]$ in $D$ not $L_{D},\left[p^{\prime} ; q^{\prime}\right]$ denotes $[l ; p]$ in $D$, and $D^{o}=D-\left\{L_{D}\right\}$.

Definition 3.2 Assume the setting of Definition $3.1, h$ is in $\mathbb{R}[u ; v], B$ is a real-valued function defined on $D$, and $G$ is a function from $\mathbb{R}$ into $\mathbb{R}$. Then $\sum_{D^{\circ}} h G(\Delta B)$ denotes $\sum_{D^{\circ}} h(p) G\left(B[p ; q]-B\left[p^{\prime} ; q^{\prime}\right]\right)$. 
Observation 3.1 Suppose that $k<l<m, D_{1} \ll\{[k ; l]\}, D_{2} \ll\{[l ; m]\}, D_{1}$ and $D_{2}$ each contain two elements, $h$ is in $\mathbb{R}[k ; m], B$ is a real-valued function defined on $D_{1} \cup D_{2}$, and $G$ is a function from $\mathbb{R}$ into $\mathbb{R}$. Then

$$
\sum_{\left[D_{1} \cup D_{2}\right]^{o}} h G(\Delta B)=\sum_{D_{1}^{o}} h G(\Delta B)+h(l) G\left(B\left(L_{D_{2}}\right)-B\left(R_{D_{1}}\right)\right)+\sum_{D_{2}^{o}} h G(\Delta B) .
$$

Observation 3.2 Assume the setting of Definition 3.2. Then

$$
\sum_{D}[h(q)-h(q)] B[p ; q]=h(v) B\left(R_{D}\right)-h(u) B\left(L_{D}\right)-\sum_{D^{o}} h \Delta B .
$$

Theorem 3.3 Suppose the hypothesis of Theorem 3.1. Then there is $D \ll$ $\{[a ; b]\}$ such that $D$ contains two elements and $\left\{\sum_{E^{o}}|\Delta A|: E \ll D\right\}$ is bounded.

Proof. From Theorem 3.2 it follows that there is $K^{*} \geq 0$ and $D^{*} \ll\{[a ; b]\}$ such that if $I$ is in $H \ll D^{*}$, then $|A(I)| \leq K^{*}$. Suppose that the conclusion is not true. Then there is a set $M$ such that $x$ is in $M$ iff $a<x \leq b$ and if $D \ll\{[a ; x]\}$ and $D$ contains two elements, then $\left\{\sum_{E^{o}}|\Delta A|: E \ll D\right\}$ is unbounded. Note that if $x$ is in $M$ and $x<y \leq b$, then $y$ is in $M$. Let $z$ denote $\inf M . a \leq z$, and $z$ is in $M$ or not.

Suppose that $z$ is not in $M$. Then $z<b$. Suppose that $z<y<b$. There is $x$ in $M$ such that $z<x<y$, so that $y$ is in $M$. Therefore, if $D \ll\{[a ; y]\}$ and contains two elements, then $\left\{\sum_{E^{\circ}}|\Delta A|: E \ll D\right\}$ is unbounded. If $z=a$, then, if $D \ll\{[a ; y]\}$ and contains two elements, then $\left\{\sum_{E^{o}}|\Delta A|: E \ll D\right\}$ is unbounded.

So, still supposing that $z$ is not in $M$, suppose that $a<z$. Then there is $P \ll\{[a ; z]\}$ such that $P$ contains two elements and $\left\{\sum_{E^{o}}|\Delta A|: E \ll\right.$ $P\}$ is bounded. Suppose that $Q \ll\{[z ; y]\}$. There is $P^{\prime} \ll P$ such that $P^{\prime} \subseteq H^{\prime} \ll D^{*}$, and there is $Q^{\prime} \ll Q$ such that $Q^{\prime} \subseteq H^{\prime \prime} \ll D^{*}$. Now, $\left\{\sum_{W^{\circ}}|\Delta A|: W \ll P^{\prime} \cup Q^{\prime}\right\}$ is unbounded. There is $K^{* *} \geq 0$ such that if $H \ll P^{\prime}$, then $\sum_{H^{o}}|\Delta A| \leq K^{* *}$. Suppose that $0<K$. There is $W \ll P^{\prime} \cup Q^{\prime}$ such that $\sum_{W^{o}}|\Delta A|>K+K^{* *}+2 K^{*}$. Let

$$
W_{1}=\left\{I: I \text { in } W, I \subseteq J \text { in } P^{\prime}\right\} \text { and } W_{2}=\left\{I: I \text { in } W, I \subseteq J \text { in } Q^{\prime}\right\}
$$


Clearly, $W_{1} \ll P^{\prime} \ll P, W_{2} \ll Q^{\prime} \ll Q$, and $W_{1} \cup W_{2}=W$. We see that

$$
\begin{aligned}
\sum_{W_{2}^{o}}|\Delta A|= & \sum_{W_{2}^{o}}|\Delta A|+\left|A\left(L_{W_{2}}\right)-A\left(R_{W_{1}}\right)\right|-\left|A\left(L_{W_{2}}\right)-A\left(R_{W_{1}}\right)\right| \\
& +\sum_{W_{1}^{o}}|\Delta A|-\sum_{W_{1}^{o}}|\Delta A| \\
= & \sum_{W^{o}}|\Delta A|-\left|A\left(L_{W_{2}}\right)-A\left(R_{W_{1}}\right)\right|-\sum_{W_{1}^{o}}|\Delta A|>K+K^{* *}+2 K^{*} \\
& -2 K^{*}-K^{* *}=K .
\end{aligned}
$$

Therefore $\left\{\sum_{E^{o}}|\Delta A|: E \ll Q\right\}$ is unbounded.

Now, suppose that $z$ is in $M$. Then $a<z$. Suppose that $a<y<z$. $y$ is not in $M$, so that there is $P \ll\{[a ; y]\}$ such that $\left\{\sum_{H^{\circ}}|\Delta A|: H \ll P\right\}$ is bounded. Suppose that $Q \ll\{[y ; z]\}$. There is $P^{\prime} \ll P$ such that $P^{\prime} \subseteq$ $H^{\prime} \ll D^{*}$ and there is $Q^{\prime} \ll Q$ such that $Q^{\prime} \subseteq H^{\prime \prime} \ll D^{*}$. $\left\{\sum_{W_{o}}|\Delta A|: W \ll\right.$ $\left.P^{\prime} \cup Q^{\prime}\right\}$ is unbounded. Except for what amounts to the interchange of $y$ and $z$, the rest of this portion of the argument proceeds as in the above paragraph, and we again have the conclusion that $\left\{\sum_{E^{o}}|\Delta A|: E \ll Q\right\}$ is unbounded.

Therefore, either:

i) $a \leq z<b$ and if $z<y<b$ and $D \ll\{[z ; y]\}$, then $\left\{\sum_{N^{\circ}}|\Delta A|: N \ll D\right\}$ is unbounded, or

ii) $a<z \leq b$ and if $a<y<z$ and $D \ll\{[y ; z]\}$, then $\left\{\sum_{N^{o}}|\Delta A|: N \ll\right.$ $D\}$ is unbounded.

We shall assume ii); the conclusion of the theorem for i) follows similarly. There is $D^{\prime} \ll D^{*}$ such that for some $w<z,[w ; z]$ is in $D^{\prime}$. Now, suppose that $0<K^{\prime}$ and $w \leq t<z$. There is $H \ll\{[t ; z]\}$ such that $H$ has at least two elements and

$$
\sum_{H^{o}}|\Delta A|>K^{\prime}+2 K^{*}
$$

Now,

$$
\max \left\{\left|A\left(L_{H}\right)\right|,\left|A\left(R_{H}\right)\right|\right\} \leq K^{*},
$$

so $H$ has at least three elements. So

$$
K^{\prime}+2 K^{*}<\sum_{H^{o}}|\Delta A|=\left[\sum_{H^{o}-\left\{R_{H}\right\}}|\Delta A|\right]_{1}+\left|A[l ; z]-A\left[l^{\prime} ; l\right]\right| \leq[]_{1}+2 K^{*},
$$

so that

$$
K^{\prime}<\sum_{H^{o}-\left\{R_{H}\right\}}|\Delta A|
$$


It therefore follows that there is a sequence $\left\{\left[p_{j} ; q_{j}\right]\right\}_{j=1}^{\infty}$ of subintervals of $[w ; z]$ and for each $j$ in $\mathbb{P}$, a subdivision $Q_{j}$ of $\left[p_{j} ; q_{j}\right]$ containing at least two elements such that:

i) For all $n$ in $\mathbb{P}, q_{n}=p_{n+1} \rightarrow z, n \rightarrow \infty$.

ii) For all $n$ in $\mathbb{P}$,

$$
(n+1)\left[\sum_{j=1}^{n}\left[\sum_{Q_{j}} 2|A[r ; s]|\right]+n\right]<\sum_{Q_{n+1}^{o}}|\Delta A|
$$

so that

$$
\sum_{j=1}^{n}\left[\sum_{Q_{j}} 2|A[r ; s]|\right]+n<\frac{1}{n+1} \sum_{Q_{n+1}^{o}}|\Delta A| .
$$

There is an element $g$ of $\mathrm{C}[a ; b] \operatorname{such}$ that $\operatorname{Rng}(g) \subseteq[-1 ; 1]$ and for each $n$ in $\mathbb{P}$ and $[r ; s]$ in $Q_{n}^{o}$,

$$
g(r)\left(A[r ; s]-A\left[r^{\prime} ; s^{\prime}\right]\right)=\frac{1}{n}\left|A[r ; s]-A\left[r^{\prime} ; s^{\prime}\right]\right| .
$$

Suppose that $0<K$ and $D \ll\{[a ; b]\}$. There is $D^{\prime} \ll D$ such that $D^{\prime} \ll D^{*}$ and for some $N$ in $\mathbb{P}$ such that $N>1,\left[p_{N} ; z\right]$ is in $D^{\prime}$. Let $E^{\prime}=D^{\prime}-\left\{\left[p_{N} ; z\right]\right\}$. There is $N^{*}$ in $\mathbb{P}$ such that

$$
N^{*}>N+1+K+4 K^{*}+2 \sum_{E^{\prime}}|A[r ; s]| .
$$

Let

$$
E^{\prime \prime}=E^{\prime} \cup\left[\cup_{j=N}^{N^{*}} Q_{j}\right] \cup\left\{\left[q_{N^{*}} ; z\right]\right\} .
$$

Clearly, $E^{\prime \prime} \ll D^{\prime}$. Now,

$$
\begin{aligned}
& \left|\sum_{E^{\prime \prime}}(g(s)-g(r)) A[r ; s]\right| \\
& =\mid \sum_{E^{\prime}}(g(s)-g(r)) A[r ; s]+\sum_{j=N}^{N^{*}-1} \sum_{Q_{j}}(g(s)-g(r)) A[r ; s] \\
& \quad+\sum_{Q_{N^{*}}}(g(s)-g(r)) A[r ; s]+\left(g(z)-g\left(q_{N^{*}}\right)\right) A\left[q_{N^{*}} ; z\right] \mid \\
& \geq\left|\sum_{Q_{N^{*}}}(g(s)-g(r)) A[r ; s]\right|-\left|\sum_{E^{\prime}}(g(s)-g(r)) A[r ; s]\right| \\
& \quad-\sum_{j=N}^{N^{*}-1} \sum_{Q_{j}}|(g(s)-g(r)) \| A[r ; s]|-\left|g(z)-g\left(q_{N^{*}}\right)\right|\left|A\left[q_{N^{*}} ; z\right]\right|
\end{aligned}
$$




$$
\begin{aligned}
\geq & \left|\sum_{Q_{N^{*}}}(g(s)-g(r)) A[r ; s]\right|-\left[2 \sum_{E^{\prime}}|A[r ; s]|+\left(\sum_{j=N}^{N^{*}-1} \sum_{Q_{j}} 2|A[r ; s]|\right)\right. \\
& \left.+2\left|A\left[q_{N^{*}} ; z\right]\right|\right]_{2} \\
= & \left|g\left(q_{N^{*}}\right) A\left(R_{Q_{N^{*}}}\right)-g\left(p_{N^{*}}\right) A\left(L_{Q_{N^{*}}}\right)-\sum_{Q_{N^{*}}^{o}} g \Delta A\right|-[]_{2} \\
\geq & \left|\sum_{Q_{N^{*}}^{o}} g \Delta A\right|-K^{*}-K^{*}-[]_{2} \\
= & \left(\sum_{Q_{N^{*}}^{o}}\left(\frac{1}{N^{*}}\right)|\Delta A|\right)-K^{*}-K^{*}-[]_{2} \\
> & \sum_{j=1}^{N^{*}-1}\left[\sum_{Q_{j}} 2|A[r ; s]|\right]+N^{*}-1-2 K^{*}-2 \sum_{E^{\prime}}|A[r ; s]| \\
& -\sum_{j=N}^{N^{*}-1}\left[\sum_{Q_{j}} 2|A[r ; s]|\right]-2 K^{*} \\
\geq & \sum_{j=N}^{N^{*}-1}\left[\sum_{Q_{j}} 2|A[r ; s]|\right]+1+K+4 K^{*}+2 \sum_{E^{\prime}}|A[r ; s]| \\
& -1-2 K^{*}-2 \sum_{E^{\prime}}|A[r ; s]|-\sum_{j=N}^{N^{*}-1}\left[\sum_{Q_{j}} 2|A[r ; s]|\right]-2 K^{*}=K,
\end{aligned}
$$

a contradiction of the hypothesis.

Therefore the conclusion of the theorem holds.

\section{Some Limit Consequences of the Boundedness of Cer- tain Sum Collections and an Integral Existence Asser- tion}

The main theorem of this section is Theorem 4.8, as stated in the introduction.

Theorem 4.1 Suppose that $a<b, A$ is an element of $\mathbb{R S I}[a ; b], D$ is a nondegenerate subdivision of $[a ; b], 0 \leq M$ and if $E \ll D$, then

$$
\sum_{E^{o}}\left|A[p ; q]-A\left[p^{\prime} ; q^{\prime}\right]\right| \leq M .
$$


Then there are elements $r^{*}$ and $l^{*}$ of $\mathbb{R}[a ; b]$ such that if $a<x \leq b$, then $A[w ; s] \rightarrow l^{*}(x)$ as $w \rightarrow x$, for $a \leq w<s<x$, and if $a \leq x<b$, then $A[w ; s] \rightarrow r^{*}(x)$ as $s \rightarrow x$, for $x<w<s \leq b$.

Proof. Clearly, it is sufficient to show convergence. We treat the case of $a<x \leq b$; the other case holds in a similar fashion. Suppose the contrary convergence assumption. There is $[u ; v]$ in $D$ such that $u<x \leq v$. It follows from routine completeness considerations that there is $K<0$ such that if $u \leq t<z<x$, then for some $[i ; j]$ for which $z<i<j<x, \mid A[t ; z]-$ $A[\bar{i} ; j] \mid \geq K$. There is $N$ in $\mathbb{P}$ such that $N>\frac{M}{K}$. It follows that there is a sequence $\left\{\left[v_{k} ; w_{k}\right]\right\}_{k=1}^{N+1}$ of subintervals of $[u ; x]$ such that for all $k$ in $\mathbb{P}$ such that $1 \leq k \leq N, w_{k} \leq v_{k+1}$ and

$$
\sum_{k=1}^{N}\left|A\left[v_{k+1} ; w_{k+1}\right]-A\left[v_{k} ; w_{k}\right]\right|>N K>M,
$$

Clearly, there is $H \ll\{[u ; x]\}$ such that $\left\{\left[v_{k} ; w_{k}\right]: k=1, \ldots, N+1\right\} \subseteq H$, and there is $E \ll D$ such that $H \subseteq E$, so that

$$
\begin{aligned}
\sum_{E^{o}}\left|A[p ; q]-A\left[p^{\prime} ; q^{\prime}\right]\right| & \geq \sum_{H^{o}}\left|A[p ; q]-A\left[p^{\prime} ; q^{\prime}\right]\right| \\
& \geq \sum_{k=1}^{N}\left|A\left[v_{k+1} ; w_{k+1}\right]-A\left[v_{k} ; w_{k}\right]\right|>M ;
\end{aligned}
$$

this contradiction establishes the theorem.

Theorem 4.2 Suppose that $a<b$ and each of $h$ and $m$ is an element of $\mathbb{R}[a ; b]$ such that:

1. $m$ is nondecreasing on $[a ; b]$.

2. For some $W>0,|\Delta h| \leq W|\Delta m|$ for all subintervals of $[a ; b]$

3. If $A$ is an element of $\mathbb{R S I}[a ; b]$ such that for each $[p ; q] \subseteq[a ; b]$,

$$
A[p ; q]=\frac{h(q)-h(p)}{m(q)-m(p)},
$$

then A satisfies the hypothesis of Theorem 4.1.

Then, there are elements $l$ and $r$ of $\mathbb{R}[a ; b]$ such that if $a<x \leq b$, then $A[w ; x] \rightarrow l(x)$ as $w \rightarrow x$, for $a \leq w<x$, and if $a \leq x<b$, then $A[x ; w] \rightarrow$ $r(x)$ as $w \rightarrow x$ for $x<w \leq b$.

Proof. Again, we need only to show convergence. We treat $a<x \leq b$; the other case follows in a similar fashion. As is well-known, $h$ and $m$ are quasi-continuous on $[a ; b]$. 
Either $m(x)-m(x-)=0$ or not. If not, then

$$
\frac{h(w)-h(x)}{m(w)-m(x)} \rightarrow \frac{h(x-)-h(x)}{m(x-)-m(x)} \text { as } w \rightarrow x, \text { for } a \leq w<x .
$$

Now, if for some $y$ such that $a \leq y<x, m(x)-m(y)=0$, then for all $w$ in $[y ; x), m(x)-m(w)=0$, so by statement 2) of the hypothesis, $h(x)-h(w)=0$, so $\frac{h(x)-h(w)}{m(x)-m(w)}=0$, so $A[w ; x] \rightarrow 0$ as $w \rightarrow x, a \leq w<x$.

So now suppose that $m(x)-m(x-)=0$ and that if $a \leq y<x$, then $m(x)-m(y) \neq 0$. Suppose that $0<c$. By Theorem 4.1 there is $t$ in $[a ; x)$ such that if $t \leq p<q<x$, then, with reference to Theorem 4.1,

$$
\left|l^{*}(x)-A[p ; q]\right|<\frac{c}{4} .
$$

Suppose that $t \leq w<x$. There is $z$ in $(w ; x)$ such that

$$
m(x)-m(z)<K=\left[\frac{m(x)-m(w)}{2}\right] \min \left\{1, \frac{c}{8(1+W)}\right\}
$$

Note that

$$
m(z)-m(w)>\frac{m(x)-m(w)}{2} .
$$

Now,

$$
\begin{aligned}
\mid & \frac{h(x)-h(w)}{m(x)-m(w)}-l^{*}(x) \mid \\
\leq & {\left[\left|\frac{(h(x)-h(z))+(h(z)-h(w))}{(m(x)-m(z))+(m(z)-m(w))}-\frac{h(z)-h(w)}{m(z)-m(w)}\right|\right]_{1} } \\
& +\left|A[w ; z]-l^{*}(x)\right| \\
< & {[\quad]_{1}+\frac{c}{4} } \\
= & \left|\frac{(h(x)-h(z))(m(z)-m(w))-(h(z)-h(w))(m(x)-m(z))}{(m(x)-m(w))(m(z)-m(w))}\right|+\frac{c}{4} \\
\leq & {\left[\frac{W(m(x)-m(z))(m(z)-m(w))+W(m(z)-m(w))(m(x)-m(z))}{(m(x)-m(w))}\right] } \\
& \times \frac{2}{(m(x)-m(w))}+\frac{c}{4} \\
\leq & 4 W K\left[\frac{(m(z)-m(w))}{(m(x)-m(w))^{2}}\right]+\frac{c}{4}
\end{aligned}
$$




$$
\begin{aligned}
& \leq 4 W\left[\frac{(m(x)-m(w))}{2}\right]\left[\frac{c}{8(1+W)}\right]\left[\frac{1}{(m(x)-m(w))}\right]+\frac{c}{4} \\
& =\left[\begin{array}{l}
c \\
4
\end{array}\right]\left[\frac{W}{1+W}\right]+\frac{c}{4}<c
\end{aligned}
$$

so the convergence assertion holds.

Theorem 4.3 Suppose that $a<b, h, m$ and $A$ are as in the hypothesis of Theorem 4.2 with $M$ as in the hypothesis of Theorem 4.1 and $l$ and $r$ as in the conclusion of Theorem 4.2. Then $l$ and $r$ have bounded variation on $[a ; b]$ and, if $a<x \leq b$, then $l(x-)=r(x-)$, and, if $a \leq x<b$, then $l(x+)=r(x+)$.

Proof. We show bounded variation on $[a ; b]$ for $l ; r$ follows similarly. Clearly, for $a<x \leq b,|l(x)| \leq W$. Suppose that $E$ is a nondegerate refinement of $D$. Let $N=$ the number of elements of $E$. By Theorem 4.2 , for each $[p ; q]$ in $E$, there is $z_{q}$ in $(p ; q)$ such that

$$
\left|A\left[z_{q} ; q\right]-l(q)\right|<\frac{1}{(N+1)}
$$

There is $[a ; y]$ in $E$ and

$$
\left\{\left[z_{v} ; v\right]: v \text { is } p \text { or } q \text { for some }[p ; q] \text { in } E^{o}\right\} \subseteq H \ll E .
$$

So

$$
\begin{aligned}
\sum_{E}|l(q)-l(p)|= & |l(y)-l(a)|+\sum_{E^{o}} \mid l(q)-A\left[z_{q} ; q\right]+A\left[z_{q} ; q\right]-A\left[z_{p} ; p\right] \\
& +A\left[z_{p} ; p\right]-l(p) \mid \\
\leq & |l(a)|+W+\sum_{E^{o}}\left|l(q)-A\left[z_{q} ; q\right]\right|+\sum_{E^{o}} \mid A\left[z_{q} ; q\right] \\
& -A\left[z_{p} ; p\right]\left|+\sum_{E^{o}}\right| A\left[z_{p} ; p\right]-l(p) \mid \\
\leq & |l(a)|+W+\frac{N}{(N+1)}+M+\frac{N}{(N+1)} \\
< & |l(a)|+W+M+2 .
\end{aligned}
$$

Therefore $l$, and similarly $r$, has bounded variation on $[a ; b]$ and so each is quasi-continuous on $[a ; b]$

We now show that if $a<x \leq b$, then $l(x-)=r(x-)$; the corresponding final equality follows similarly. Let $l^{*}$ and $r^{*}$ be as in Theorem 4.1. Suppose that $a<x \leq b$ and $0<c$. By Theorem 4.1 there is $t$ in $[a ; x)$ such that if 
$t \leq p<q<x$, then $\left|A[p ; q]-l^{*}(x)\right|<\frac{c}{4}$. Suppose that $z$ is in $(t ; x) . z$ is in some $(u ; v) \subseteq(t ; x)$ such that

$$
\max \{|A[u ; z]-l(z)|,|A[z ; v]-r(z)|\}<\frac{c}{4} .
$$

Clearly

so

$$
\max \left\{\left|A[u ; z]-l^{*}(x)\right|,\left|A[z ; v]-l^{*}(x)\right|\right\}<\frac{c}{4}
$$

$$
\begin{aligned}
& \max \left\{\left|l(z)-l^{*}(x)\right|,\left|r(z)-l^{*}(x)\right|\right\} \\
& \leq \max \left\{|l(z)-A[u ; z]|+\left|A[u ; z]-l^{*}(x)\right|,|r(z)-A[z ; v]|+\left|A[z ; v]-l^{*}(x)\right|\right\} \\
& \leq \max \left\{\frac{c}{4}+\frac{c}{4}, \frac{c}{4}+\frac{c}{4}\right\}=\frac{c}{2}<c .
\end{aligned}
$$

Therefore $l(x-)=l^{*}(x)=r(x-)$ and, in a similar fashion, $l(x+)=$ $r^{*}(x)=r(x+)$.

Theorem 4.4 Assume the hypothesis of Theorem 4.2. Suppose that $0<c$. Then there is $E \ll\{[a ; b]\}$ such that if $[p ; q]$ is in $E$, then $\min \{\mid r(p)-$ $A[p ; q]|| l,(q)-A[p ; q] \mid\}<c$.

Proof. There is $y$ in $(a ; b]$ such that $|r(a)-A[a ; y]|<c$. Therefore there is a set $Z$ such that $x$ is in $Z$ iff $a<x \leq b$ and for some subdivision $E$ of $[a ; x]$, if $[p ; q]$ is in $E$, then

$$
\min \{|r(p)-A[p ; q]|,|l(q)-A[p ; q]|\}<c .
$$

Let $t=\sup Z$. There is $s$ in $[a ; t)$ such that if $s \leq y<t$, then

$$
|A[y ; t]-l(t)|<c \text {. }
$$

There is $v$ in $Z$ such that $s \leq v$. If $v=t$, then clearly $t$ is in $Z$. So suppose that $v<t$. Then

$$
|A[v ; t]-l(t)|<c
$$

furthermore, for some subdivison $H$ of $[a ; v]$, for each $[p ; q]$ in $H$,

$$
\min \{|r(p)-A[p ; q]|,|l(q)-A[p ; q]|\}<c ;
$$

considering $H^{\prime}=H \cup\{[v ; t]\}$, we see that $t$ is in $Z$.

Now suppose, on the contrary, that $t<b$. There is a subdivision $H$ of $[a ; t]$ such that if $[p ; q]$ is in $H$, then

$$
\min \{|r(p)-A[p ; q]|,|l(q)-A[p ; q]|\}<c .
$$

There is $u$ in $(t ; b]$ such that $|r(t)-A[t ; u]|<c$. Considering $H^{\prime}=H \cup\{[t ; u]\}$, we see that $u$ is in $Z$; since $t<u$, we have a contradiction.

Therefore the theorem is true. 
Theorem 4.5 Again, assume the hypothesis of Theorem 4.2. Then there is an element $\alpha$ of $\mathbb{R S I}[a ; b]$ such that if $a \leq p<q \leq b$, then $\alpha[p ; q]$ is $r(p)$ or $l(q)$, and if $a \leq u<v \leq b$, then the following existence and equality holds:

$$
\left.h\right|_{u} ^{v}=\int_{[u ; v]} \alpha(I) d m
$$

Proof. Let $\gamma_{r}$ and $\gamma_{l}$ denote the elements of $\mathbb{R S I}[a ; b]$ given by

$$
\gamma_{r}[p ; q]=r(p) \text { and } \gamma_{l}[p ; q]=l(q) .
$$

By virtue of the quasi-continuity of $r$ and $l$ and the fact that $m$ is nondecreasing, it is well-known and easily shown that if $a \leq p<q \leq b$ and $\gamma$ is $\gamma_{r}$ or $\gamma_{l}$, then:

1) $\int_{[p ; q]} \gamma(I) d m$ exists, and

2) if $W$ is an element of $\mathbb{R}[a ; b]$ such that for $a \leq p<q \leq b, W(q)-W(p)=$ $\int_{[p ; q]} \gamma(I) d m$, then $W$ is in $\mathrm{BV}[a ; b]$.

From Theorem 2.A.2 we have, for $a \leq u<v \leq b$, the following succession of existence and equivalence assertions:

$$
\begin{aligned}
\int_{[u ; v]} & \min \left\{\int_{I}\left|\int_{J} \gamma_{r} d m-d h\right|, \int_{I}\left|\int_{J} \gamma_{l} d m-d h\right|\right\} \\
= & \int_{[u ; v]} \min \left\{\int_{I}\left|\gamma_{r} d m-d h\right|, \int_{I}\left|\gamma_{l} d m-d h\right|\right\} \\
= & \int_{[u ; v]} \min \left\{\left|\gamma_{r} d m-d h\right|,\left|\gamma_{l} d m-d h\right|\right\} .
\end{aligned}
$$

Clearly, there is an element $\alpha$ of $\mathbb{R S I}[a ; b]$ such that if $a \leq p<q \leq b$, then

$$
\begin{aligned}
\alpha[p ; q] & =\gamma_{r}[p ; q] \text { or } \gamma_{l}[p ; q], \text { and } \\
|\alpha[p ; q] m|_{p}^{q}-\left.h\right|_{p} ^{q} \mid & =\min \left\{\left|\gamma_{r}[p ; q] m\right|_{p}^{q}-\left.\left.h\right|_{p} ^{q}|,| \gamma_{l}[p ; q] m\right|_{p} ^{q}-\left.h\right|_{p} ^{q} \mid\right\}
\end{aligned}
$$

so that from (4.5.1) it immediately follows that if $a \leq u<v \leq b$, then

$$
\int_{[u ; v]}|\alpha(I) d m-d h|
$$

exists.

We now show that if $a \leq u<v \leq b$, then

$$
\int_{[u ; v]}|\alpha(I) d m-d h|=0 .
$$


Suppose that $0<c$. There is $D \ll\{[u ; v]\}$ such that if $E \ll D$, then

$$
\left|\int_{[u ; v]}\right| \alpha(I) d m-d h\left|-\sum_{E}\right| \alpha \Delta_{I} m-\Delta_{I} h||<\frac{c}{2} .
$$

By Theorem 4.4, for each $[p ; q]$ in $D$, there is $E[p ; q] \ll\{[p ; q]\}$ such that if $J$ is in $E[p ; q]$, then, from (4.5.2),

$$
\left|\alpha(J)-\frac{\Delta_{J} h}{\Delta_{J} m}\right|=\min \left\{\left|\gamma_{r}(J)-\frac{\Delta_{J} h}{\Delta_{J} m}\right|,\left|\gamma_{l}(J)-\frac{\Delta_{J} h}{\Delta_{J} m}\right|\right\}<\frac{c}{2\left(1+\left.m\right|_{u} ^{v}\right)},
$$

so that

$$
\begin{aligned}
\int_{[u ; v]}|\alpha(I) d m-d h| \leq \mid & \int_{[u ; v]}|\alpha(I) d m-d h|-\sum_{D} \sum_{E[p ; q]}\left|\alpha(J) \Delta_{J} m-\Delta_{J} h\right| \mid \\
& +\sum_{D} \sum_{E[p ; q]}\left|\alpha(J) \Delta_{J} m-\Delta_{J} h\right| \\
& <\frac{c}{2}+\sum_{D} \sum_{E[p ; q]}\left|\alpha(J)-\frac{\Delta_{J} h}{\Delta_{J} m}\right| \Delta_{J} m \\
\leq & \frac{c}{2}+\sum_{D} \sum_{E[p ; q]}\left[\frac{c}{2\left(1+\left.m\right|_{u} ^{v}\right)}\right] \Delta_{J} m \\
= & \frac{c}{2}+\left(\frac{c}{2}\right)\left(\frac{\left.m\right|_{u} ^{v}}{\left(1+\left.m\right|_{u} ^{v}\right)}\right)<\frac{c}{2}+\left(\frac{c}{2}\right)=c .
\end{aligned}
$$

Therefore

$$
\int_{[u ; v]}|\alpha(I) d m-d h|=0,
$$

which, by routine considerations, implies that

$$
\left.h\right|_{u} ^{v}=\int_{[u ; v]} \alpha(I) d m .
$$

Theorem 4.6 Assume the hypothesis of Theorem 4.2. Suppose further that $w$ is in $\mathbb{R}[a ; b], c$ is in $[a ; b]$ and either:

a) $a \leq c<b$ and for all $x$ in $[a ; b], w(x)=0$ if $a \leq x \leq c$, and $w(x)=1$ if $c<x \leq b$, or

b) $a<c \leq b$ and for all $x$ in $[a ; b], w(x)=0$ if $a \leq x<c$, and $w(x)=1$ if $c \leq x \leq b$, or

c) $w(x)=1$ if $a \leq x \leq b$. 
Then

$$
\int_{[a ; b]} \frac{d w d h}{d m}
$$

exists.

INDICATION OF PROOF. In each case, the verification of the convergence assertion in very easy; we state the value of the integral and leave the details to the reader: a) $r(c)$. b) $l(c)$. c) 0 .

Theorem 4.7 Assume the hypothesis of Theorem 4.2. Suppose further that $g$ is in $\mathbb{R}[a ; b], P \ll\{[a ; b]\}$ and if $[p ; q]$ is in $P$ and $p<x_{1}<x_{2}<q$, then $g\left(x_{1}\right)=g\left(x_{2}\right)$. Then

$$
\int_{[a ; b]} \frac{d g d h}{d m}
$$

exists.

INDICATION OF PROOF. $g$ is a finite linear combination on $[a ; b]$ of functions, each of which satisfies a), b) or c) of the hypothesis of Theorem 4.6.

Theorem 4.8 Assume the hypothesis of Theorem 4.2. Suppose further that $k$ is in $\mathbb{R}[a ; b]$, quasi-continuous on $[a ; b]$. Then

$$
\int_{[a ; b]} \frac{d k d h}{d m}
$$

exists.

IndicAtion OF PROOF. Suppose that $0<c$. There is $g$ satisfying the hypothesis of Theorem 4.7 such that

$$
|k(x)-g(x)|<\frac{c}{(1+2 W+M)}, a \leq x \leq b .
$$

Let $f=k-g$. Suppose that $H \ll\{[a ; b]\}$ and $H$ is nondegenerate. Then (see Definition 3.1 through Observation 3.2)

$$
\begin{aligned}
& \left|\sum_{H}(k(q)-k(p)) A[p ; q]-\sum_{H}(g(q)-g(p)) A[p ; q]\right|=\left|\sum_{H}(f(q)-f(p)) A[p ; q]\right| \\
& \quad=\left|f(b) A\left(R_{H}\right)-f(a) A\left(L_{H}\right)-\sum_{H^{o}} f(p)\left[A[p ; q]-A\left[p^{\prime} ; q^{\prime}\right]\right]\right| \\
& \quad \leq|f(b)|\left|A\left(R_{H}\right)\right|+|f(a)|\left|A\left(L_{H}\right)\right|+\sum_{H^{o}}|f(p)|\left|A[p ; q]-A\left[p^{\prime} ; q^{\prime}\right]\right| \\
& \quad<\left(\frac{c}{(1+2 W+M)}\right)[W+W+M]<c .
\end{aligned}
$$

Theorem 4.7 and routine completeness considerations complete the proof. 


\section{A Convexity Decomposition Theorem}

In this section we prove Theorem 5.4, as stated in the introduction.

We begin with a special integral existence theorem.

Theorem 5.1 Suppose that $a<b$ and each of $f$ and $g$ is in $\operatorname{BV}(\mathbb{R})[a ; b]$. Suppose that $B$ is an element of $\mathbb{R S I}[a ; b]$ given by

$$
B(I)=\sup \{|j(x)-k(y)|:(x, y) \text { in } I \times I,(j, k) \text { in }\{f, g\} \times\{f, g\}\} .
$$

Then the following statements are true:

1) If $[u ; v] \subseteq[a ; b]$ and $E \ll D \ll\{[u ; v]\}$, then

$$
\sum_{D} B(I) \leq \sum_{E} B(J)
$$

so that

$$
\int_{[u ; v]} B(I) \text { exists iff }\left[\sup \left\{\sum_{H} B(J): H \ll\{[u ; v]\}\right\}\right]_{1}<\infty
$$

in which case

$$
\int_{[u ; v]} B(I)=[\quad]_{1}
$$

2) If, for each $x$ in $[a ; b], f(x+)=g(x+)$ if $a \leq x<b$ and $f(x-)=g(x-)$ if $a<x \leq b$, then $\int_{[a ; b]} B(I)$ exists.

Proof. We shall first indicate a proof of (5.1.1) by showing a simple inequality and leaving the induction to the reader.

Suppose that $a \leq p<q<s \leq b$. Suppose that $0<c$. There are $x$ and $y$ such that $p \leq x \leq y \leq s$ and $(j, k)$ in $\{f, g\} \times\{f, g\}$ such that

$$
0 \leq B[p ; s]-|j(y)-k(x)|<c .
$$

If $p \leq x \leq y \leq q$ or $q \leq x \leq y \leq s$, then

$$
\begin{aligned}
B[p ; s] & <|j(y)-k(x)|+c \leq \max \{B[p ; q], B[q ; s]\}+c \leq B[p ; q]+B[q ; s]+c . \\
\quad \text { If } p & \leq x \leq q \leq y \leq s, \text { then } \\
B[p ; s] & <|j(y)-k(x)|+c \leq|j(y)-j(q)|+|j(q)-k(x)|+c \leq B[q ; s]+B[p ; q]+c .
\end{aligned}
$$

In either case,

$$
B[p ; s] \leq B[p ; q]+B[q ; s]+c .
$$


Therefore

$$
B[p ; s] \leq B[p ; q]+B[q ; s] .
$$

As stated above, (5.1.1) follows from (5.1.4) by induction; we let the reader furnish this as well as the routine arguments for (5.1.2) and (5.1.3).

Now, suppose the hypothesis of 2). It is well-known and easily shown that

$$
\sup \left\{\sum_{W}|f(z)-g(z)|: W \text { a finite subset of }[a ; b]\right\}=S<\infty .
$$

Suppose that $a \leq p \leq x \leq y \leq q \leq b$ and $(j, k)$ is in $\{f, g\} \times\{f, g\}$. Then

$$
\begin{aligned}
|j(x)-k(y)| & \leq|j(x)-j(y)|+|j(y)-k(y)| \\
& \leq \max \left\{\int_{[p ; q]}|d f|, \int_{[p ; q]}|d g|\right\}+|f(y)-g(y)| .
\end{aligned}
$$

So now suppose that $H \ll\{[a ; b]\}$. Let $N=$ the number of elements of $H$. For each $[p ; q]$ in $H$ there is $x$ and $y$ such that $p \leq x \leq y \leq q$ and $(j, k)$ in $\{f, g\} \times\{f, g\}$ such that

$$
0 \leq B[p ; q]-|j(x)-k(y)|<\frac{1}{N},
$$

so that

$$
\begin{aligned}
\sum_{H} B[p ; q] & <\sum_{H}\left[|j(x)-k(y)|+\frac{1}{N}\right] \\
& \leq \sum_{H}\left[\max \left\{\int_{[p ; q]}|d f|, \int_{[p ; q]}|d g|\right\}+|f(y)-g(y)|\right]+1 \\
& \leq \sum_{H}\left[\int_{[p ; q]}|d f|+\int_{[p ; q]}|d g|\right]+\sum_{H}|f(y)-g(y)|+1 \\
& \leq \int_{[a ; b]}|d f|+\int_{[a ; b]}|d g|+2 S+1 .
\end{aligned}
$$

It therefore follows that $\int_{[a ; b]} B(I)$ exists.

Theorem 5.2 Assume the setting of Theorem 4.3, and B defined for $l$ and $r$ as for $f$ and $g$ as in Theorem 5.1. The following statements are true:

1) From Theorem 4.3 it follows that additionally the hypothesis of 2) of Theorem 5.1 holds, so that $\int_{[a ; b]} B(I)$ exists. 
2) Let $V$ denote the element of $\mathbb{R}[a ; b]$ given by:

$$
V(a)=0, \text { and, if } a<x \leq b, \text { then } V(x)=\int_{[a ; x]} B(I) .
$$

Then, if $a \leq p<q<s \leq b$, and $0<\min \left\{\left.m\right|_{p} ^{q},\left.m\right|_{q} ^{s}\right\}$, then

$$
(V(q)+V(p))-\frac{\left.h\right|_{p} ^{q}}{\left.m\right|_{p} ^{q}} \leq(V(s)+V(q))-\frac{\left.h\right|_{q} ^{s}}{\left.m\right|_{q} ^{s}} .
$$

Proof. 1) is immediate.

Consider 2) Suppose that $0<c$. By Theorem 4.5 and with $\alpha$ as in that theorem, it follows from some elementary inequality calculations that there are subdivisions $D_{1}$ and $D_{2}$ of $[p ; q]$ and $[q ; s]$, respectively, such that

$$
\left|\frac{\left.h\right|_{p} ^{q}}{\left.m\right|_{p} ^{q}}-\frac{\sum_{D_{1}} \alpha(I) m(I)}{\left.m\right|_{p} ^{q}}\right|<\frac{c}{2} \text { and }\left|\frac{\left.h\right|_{q} ^{s}}{\left.m\right|_{q} ^{s}}-\frac{\sum_{D_{2}} \alpha(I) m(I)}{\left.m\right|_{q} ^{s}}\right|<\frac{c}{2} .
$$

Now,

$$
\begin{aligned}
(V(s)+ & V(q))-\frac{\left.h\right|_{q} ^{s}}{\left.m\right|_{q} ^{s}}-\left[(V(q)+V(p))-\frac{\left.h\right|_{p} ^{q}}{\left.m\right|_{p} ^{q}}\right] \\
= & (V(s)-V(p))-\left[\frac{\left.h\right|_{q} ^{s}}{\left.m\right|_{q} ^{s}}-\frac{\left.h\right|_{p} ^{q}}{\left.m\right|_{p} ^{q}}\right] \\
= & \int_{[p ; s]} B+\frac{\sum_{D_{2}} \alpha(I) m(I)}{\left.m\right|_{q} ^{s}}-\frac{\left.h\right|_{q} ^{s}}{\left.m\right|_{q} ^{s}}+\frac{\left.h\right|_{p} ^{q}}{\left.m\right|_{p} ^{q}}-\frac{\sum_{D_{1}} \alpha(I) m(I)}{\left.m\right|_{p} ^{q}} \\
& \quad-\frac{\sum_{D_{2}} \alpha(I) m(I)}{\left.m\right|_{q} ^{s}}+\frac{\sum_{D_{1}} \alpha(I) m(I)}{\left.m\right|_{p} ^{q}} \\
\geq & \int_{[p ; s]} B(I)-\frac{c}{2}-\frac{c}{2}-\max \left\{\alpha(I): I \operatorname{in} D_{2}\right\}+\min \left\{\alpha(I): I \text { in } D_{1}\right\} \\
\geq & B[p ; s]-\mid \max \left\{\alpha(I): I \text { in } D_{2}\right\}-\min \left\{\alpha(I): I \text { in } D_{1}\right\} \mid-c \geq-c .
\end{aligned}
$$

It therefore follows that

$$
(V(s)+V(q))-\frac{\left.h\right|_{q} ^{s}}{\left.m\right|_{q} ^{s}}-\left[(V(q)+V(p))-\frac{\left.h\right|_{p} ^{q}}{\left.m\right|_{p} ^{q}}\right] \geq 0 ;
$$

this establishes the conclusion of 2).

Corollary 5.1 Assume the setting of Theorem 5.2. If $a \leq t<u \leq w<v \leq b$ and $0<\min \left\{\left.m\right|_{t} ^{u},\left.m\right|_{w} ^{v}\right\}$, then

$$
V(u)+V(t)-\frac{\left.h\right|_{t} ^{u}}{\left.m\right|_{t} ^{u}} \leq V(v)+V(w)-\frac{\left.h\right|_{w} ^{v}}{\left.m\right|_{w} ^{v}}
$$


ProOF. Either $0<\left.m\right|_{u} ^{w}$ or not.

In the first case, $u<w$, so by Theorem 5.2,

$$
\begin{aligned}
V(u)+V(t)-\frac{\left.h\right|_{t} ^{u}}{\left.m\right|_{t} ^{u}} & \leq V(w)+V(u)-\frac{\left.h\right|_{u} ^{w}}{\left.m\right|_{u} ^{w}} \\
& \leq V(v)+V(w)-\frac{\left.h\right|_{w} ^{v}}{\left.m\right|_{w} ^{v}}
\end{aligned}
$$

so in this case the conclusion holds.

We note that $V$ is nondecreasing on $[a ; b]$.

Now, suppose that $0=\left.m\right|_{u} ^{w}$. Then, the final inequality below due to Theorem 5.2,

$$
\begin{aligned}
V(u)+V(t)-\frac{\left.h\right|_{t} ^{u}}{\left.m\right|_{t} ^{u}} & =V(u)+V(t)-\frac{\left.h\right|_{t} ^{w}}{\left.m\right|_{t} ^{w}} \\
& \leq V(w)+V(t)-\frac{\left.h\right|_{t} ^{w}}{\left.m\right|_{t} ^{w}} \leq V(v)+V(w)-\frac{\left.h\right|_{v} ^{w}}{\left.m\right|_{v} ^{w}}
\end{aligned}
$$

so that in this case the conclusion holds.

Before we prove the decomposition theorem given in the introduction, we state a simple inequality lemma.

Lemma 5.1 Suppose that $a<b, m$ is an element of $\mathbb{R}[a ; b]$, nondecreasing on $[a ; b]$ and $\alpha$ is an element of $\mathbb{R S I}[a ; b]$ such that if $a \leq i<j \leq k<l \leq b$ and $0<\min \left\{\left.m\right|_{i} ^{j},\left.m\right|_{k} ^{l}\right\}$, then $\alpha[i ; j] \leq \alpha[k ; l]$. Then, if $a \leq t<u \leq w<v \leq b$, $0<\min \left\{\left.m\right|_{t} ^{u},\left.m\right|_{w} ^{v}\right\}, D_{1} \ll\{[t ; u]\}$ and $D_{2} \ll\{[w ; v]\}$, then

$$
\frac{\sum_{D_{1}} \alpha(I) \Delta_{I} m}{\left.m\right|_{t} ^{u}} \leq \frac{\sum_{D_{2}} \alpha(I) \Delta_{I} m}{\left.m\right|_{w} ^{v}} ;
$$

in particular, if $\int_{[t ; u]} \alpha d m$ and $\int_{[w ; v]} \alpha d m$ exist, then

$$
\frac{\int_{[t ; u]} \alpha d m}{\left.m\right|_{t} ^{u}} \leq \frac{\int_{[w ; v]} \alpha d m}{\left.m\right|_{w} ^{v}} .
$$

INDICATION OF PROOF. Letting

$g_{1}=\max \left\{\alpha(I): I\right.$ in $\left.D_{1}, \Delta_{I} m \neq 0\right\}$ and $g_{2}=\min \left\{\alpha(I): I\right.$ in $\left.D_{2}, \Delta_{I} m \neq 0\right\}$,

we see that

$$
\begin{aligned}
\frac{\sum_{D_{1}} \alpha(I) \Delta_{I} m}{\left.m\right|_{t} ^{u}} & \leq \frac{\sum_{D_{1}} g_{1} \Delta_{I} m}{\left.m\right|_{t} ^{u}}=\frac{\left.g_{1} m\right|_{t} ^{u}}{\left.m\right|_{t} ^{u}}=g_{1} \leq g_{2} \\
& =\frac{\left.g_{2} m\right|_{t} ^{u}}{\left.m\right|_{t} ^{u}}=\frac{\sum_{D_{1}} g_{2} \Delta_{I} m}{\left.m\right|_{t} ^{u}} \leq \frac{\sum_{D_{2}} \alpha(I) \Delta_{I} m}{\left.m\right|_{t} ^{u}}
\end{aligned}
$$


The next theorem consists of a sequence of statements which, except for 5b-i) below, on the basis of the preceding results, require a treatment sufficiently routine that we can leave matters to the reader.

Theorem 5.3 Assume the setting of Theorem 5.2, with $V$ as in statement 2) of the conclusion. Let $C_{1}$ and $C_{2}$ denote elements of $\mathbb{R S I}[a ; b]$ given, respectively, by:

$$
C_{1}[p ; q]=V(q)+V(p), C_{2}[p ; q]=C_{1}[p ; q]-\frac{\left.h\right|_{p} ^{q}}{\left.m\right|_{p} ^{q}}
$$

The following statements are true:

1) $V$ is nondecreasing on $[a ; b]$, so, as is well-known, for each $[u ; v] \subseteq[a ; b]$, $\int_{[u ; v]} C_{1} d m$ exists.

2) $C_{1}$ clearly satisfies the ( $\alpha$ ) hypothesis of Lemma 5.1 .

3) By Corollary 5.2, $C_{2}$ satisfies the ( $\alpha$ ) hypothesis of Lemma 5.1.

4) If $a \leq u<v \leq b$, then $\int_{[u ; v]} C_{2} d m$ exists and is $\int_{[u ; v]} C_{1} d m-\left.h\right|_{u} ^{v}$.

5) Suppose that $f_{1}$ and $f_{2}$ are elements of $\mathbb{R}[a ; b]$ such that $f_{1}(a)=f_{2}(a)=$ 0 and, if $i=1,2$ and $a<x \leq b$, then $f_{i}(x)=\int_{[a ; x]} C_{i} d m$. Then,

a) if $a \leq x \leq b$, then $f_{1}(x)-f_{2}(x)=h(x)-h(a)$, and

b) if $i=1,2$, then $f_{i}$ is in $\operatorname{Lip}(m)[a ; b]$ and

i) for some $M_{i} \geq 0$, nondegenerate subdivision $D_{i}$ of $[a ; b]$ and each refinement $E$ of $D_{i}$,

$$
\sum_{E^{o}}\left|\frac{f_{i}(q)-f_{i}(p)}{m(q)-m(p)}-\frac{f_{i}\left(q^{\prime}\right)-f_{i}\left(p^{\prime}\right)}{m\left(q^{\prime}\right)-m\left(p^{\prime}\right)}\right| \leq M_{i}
$$

notation as before, and

ii) from Lemma $5.1, f_{i}$ is " $m$ - convex upward on $[a ; b]$ ", i.e., if $a \leq$ $t<u \leq w<v \leq b$ and $0<\min \left\{\left.m\right|_{t} ^{u},\left.m\right|_{w} ^{v}\right\}$, then

$$
\frac{\left.f_{i}\right|_{t} ^{u}}{\left.m\right|_{t} ^{u}} \leq \frac{\left.f_{i}\right|_{w} ^{v}}{\left.m\right|_{w} ^{v}}
$$

Proof OF 5-b-i). We first show that the statement holds for $f_{1}$. Suppose that $E$ is a nondegenerate subdivision of $[a ; b]$. Then

$$
\sum_{E^{o}}\left|\frac{f_{1}(q)-f_{1}(p)}{m(q)-m(p)}-\frac{f_{1}\left(q^{\prime}\right)-f_{1}\left(p^{\prime}\right)}{m\left(q^{\prime}\right)-m\left(p^{\prime}\right)}\right|=\sum_{E^{o}}\left|\frac{\int_{[p ; q]} C_{1} d m}{\left.m\right|_{p} ^{q}}-\frac{\int_{\left[p^{\prime} ; q^{\prime}\right]} C_{1} d m}{\left.m\right|_{p^{\prime}} ^{q^{\prime}}}\right|
$$




$$
\begin{aligned}
& =\sum_{E^{o}}\left[\max \left\{\frac{\int_{[p ; q]} C_{1} d m}{\left.m\right|_{p} ^{q}}, \frac{\int_{\left[p^{\prime} ; q^{\prime}\right]} C_{1} d m}{\left.m\right|_{p^{\prime}} ^{q^{\prime}}}\right\}\right. \\
& \left.\quad-\min \left\{\frac{\int_{[p ; q]} C_{1} d m}{\left.m\right|_{p} ^{q}}, \frac{\int_{\left[p^{\prime} ; q^{\prime}\right]} C_{1} d m}{\left.m\right|_{p^{\prime}} ^{q^{\prime}}}\right\}\right] \\
& \leq \sum_{E^{o}}\left[2 V(q)-2 V\left(p^{\prime}\right)\right]=2 \sum_{E^{o}}\left[V(q)-V(p)+V\left(q^{\prime}\right)-V\left(p^{\prime}\right)\right] \\
& =2\left(\sum_{E^{o}}[V(q)-V(p)]+\sum_{E^{o}}\left[V\left(q^{\prime}\right)-V\left(p^{\prime}\right)\right]\right) \\
& \leq 2(V(b)-V(a)+V(b)-V(a))=4(V(b)-V(a)) .
\end{aligned}
$$

To see that the statement holds for $f_{2}$, we need only note that for $a \leq x \leq b$,

$$
f_{2}(x)=f_{1}(x)-[h(x)-h(a)]
$$

clearly the desired conclusion follows from a routine application of the triangle inequality.

Let us note that it follows immediately from Theorem 5.3 that for Theorem 5.4,1) implies 2). With regard to showing that for Theorem 5.4, 2) implies 1), we note that given 2), for $a \leq x \leq b$

$$
h(x)=f_{1}(x)-f_{2}(x)+h(a) ;
$$

again, a routine application of the triangle inequality gives the desired conclusion.

\section{References}

[1] W. D. L. Appling, Fields of sets, set functions, set function integrals, and finite additivity, Internat. J. Math. \& Math. Sci., 7(2) (1984), 209-233, (expository paper).

[2] E. Hellinger, Die Orthogonalivarianten Quadratischer Formen von Unendlich Vielen Variablen, Diss, Gottingen, 1907.

[3] H. S. Kaltenborn, Linear functional operations on functions having discontinuities of the first kind, Bull. Amer. Math. Soc., 40 (1934), 702-708.

[4] A. N. Kolmogoroff, Untersuchungen über den integralbegriff, Math. Ann., 103 (1930), 654-696.

[5] R. E. Lane, The integral of a function with respect to a function, Proc. Amer. Math. Soc., 5 (1954), 59-66. 
[6] J. A. Reneke, Linear functionals on the space of quasi-continuous functions, Bull. Amer. Math. Soc., 72 (1966), 1023-1025.

[7] J. R. Webb, A Hellinger integral representation for bounded linear functionals, Doctoral Dissertation, University of Texas, Austin, 1960. 